\title{
Practical implementation of three-dimensional poststack depth migration
}

\author{
Moshe Reshef* and David Kesslert
}

\begin{abstract}
This work deals with the practical aspects of threedimensional (3-D) poststack depth migration. A method, based on depth extrapolation in the frequency domain, is used for the migration. This method is suitable for structures with arbitrary velocity variation, and the number of computations required can be directly related to the complexity of the given velocity function. We demonstrate the superior computational efficiency of this method for 3-D depth migration relative to the reverse-time migration method. The computational algorithm used for the migration is designed for a multiprocessor machine (Cray-XMP/48) and takes advantage of advanced disk technologies to overcome the input/output $(\mathrm{I} / \mathrm{O})$ problem. The method is demonstrated with both synthetic and field data. The migration of a typical 3-D data volume can be accomplished in only a few hours.
\end{abstract}

\section{INTRODUCTION}

The recent growth in three-dimensional (3-D) data processing leads to a need for high-quality 3-D migration algorithms. Since 3-D data processing is considered expensive, economical restrictions may dictate the use of simple and fast migration techniques such as the $f-k$ method (Stolt, 1978). Accurate prestack and poststack depth migration algorithms that can handle complicated two-dimensional (2-D) geologic structures have been developed in the last few years. However, most of these techniques are time-consuming, and although extension of these algorithms to the 3-D case is straightforward, the high cost prevents the use of these algorithms in a standard 3-D data processing sequence.

High cost is not the only obstacle to using the more accurate depth migration algorithms. In order to obtain an accurate image of the earth's subsurface, the velocity must be known in great detail. Unfortunately, this is rarely the case and therefore the justification for using these techniques is debatable. A practical solution to this dilemma is an iterative procedure of depth migration (Whitmore, 1983). In that procedure, the migration results are used to improve the velocity function. With good interpretation of the intermediate results, the final depth section can be obtained with a reasonable number of iterations. The major drawback of such methods is that the same amount of computation is required for each iteration regardless of the complexity of the velocity function.

Accurate depth migration algorithms require extrapolation of the entire wave field. This extrapolation can be done either in time or in depth (Tarantola et al., 1988). Although correct extrapolation must be based on a two-way wave equation (Kosloff and Baysal, 1983; Wapenaar and Berkhout, 1986), a one-way wave equation provides adequate accuracy for the migration of stacked data. Since methods based on these different approaches all perform well in the 2-D case, the choice of method to be implemented in three dimensions is made according to the practical aspects of each method.

The enormous amount of data and computations involved in applications such as 3-D depth migration requires that special attention be given to the computer algorithm design. When misused, even advanced supercomputers are unable to handle such large problems. The feasibility of using these computationally intensive migration techniques depends on the ability to design an efficient "out-of-core" solution. Proper data organization, along with the use of advanced storage devices and high-speed $\mathrm{I} / \mathrm{O}$ channels, helps alleviate the $\mathrm{I} / \mathrm{O}$ problem, whereas a reduction in elapsed computation time must be accomplished by the implementation of parallel processing.

In the following, we describe a 3-D poststack migration method based on a two-way wave equation. The method belongs to the space-frequency group of migrations (Berkhout, 1981) and emphasis is given to its practical aspects and the computer algorithm employed. The algorithm enables the cost of the migration to be related to the complexity of the given velocity function without affecting the accuracy. The method is compared to other migration methods and, in particular, to reverse-time migration. Finally the algorithm is tested on synthetic and field data examples.

Manuscript recejved by the Editor February 10, 1988; revised manuscript received August 12, 1988.

*Department of Geophysics and Planetary Sciences, Tel-Aviv University, Ramat-Aviv, Tel-Aviv 69978, Israel; also Cray Research, Inc., 5847 San Felipe, Houston, TX 77057.

$\$$ Department of Geophysics and Planetary Sciences, Tel-Aviv University, Ramat-Aviv, Tel-Aviv 69978, Israel.

(1989 Society of Exploration Geophysicists. All rights reserved. 


\section{MATHEMATICAL ALGORITHM}

We describe below the extension of the generalized phaseshift method (Kosloff and Kessler, 1987) to 3-D problems. The depth migration is based on the temporally transformed nonreflecting wave equation (Baysal et al., 1984) given by

$$
c \frac{\partial}{\partial x}\left(c \frac{\partial \tilde{\boldsymbol{P}}}{\partial x}\right)+c \frac{\partial}{\partial y}\left(c \frac{\partial \tilde{P}}{\partial y}\right)+c \frac{\partial}{\partial z}\left(c \frac{\partial \tilde{P}}{\partial z}\right)=-\omega^{2} \tilde{P},
$$

where $x, y$, and $z$ denote Cartesian coordinates; $c(x, y, z)$ is half the acoustic velocity; $\omega$ is the temporal frequency; and $\tilde{P}(x, y, z, \omega)$ is the transformed pressure field.

As in the 2-D case, we can recast equation (1) as a coupled first-order system given by

$$
\frac{\partial}{\partial z}\left(\begin{array}{c}
\tilde{P} \\
c \frac{\partial \tilde{P}}{\partial z}
\end{array}\right)=\left[\begin{array}{cc}
0 & \frac{1}{c} \\
-\left[\frac{\omega^{2}}{c}+\frac{\partial}{\partial x}\left(c \frac{\partial}{\partial x}\right)+\frac{\partial}{\partial y}\left(c \frac{\partial}{\partial y}\right)\right] & \\
0
\end{array}\right]\left(\begin{array}{c}
\tilde{P} \\
\partial \tilde{P} \\
c \frac{\partial}{\partial z}
\end{array}\right) .
$$

Designating the matrix on the right-hand side of equation (2) by $\mathbf{B}$, the system can be written as

$$
\frac{\partial}{\partial z} \mathbf{V}=\mathbf{B} \mathbf{V}
$$

If $N_{x}$ and $N_{y}$ denote the number of seismic traces in the $x$ and $y$ directions, respectively, the vector $\mathbf{V}$ has $2 N_{x} N_{y}$ elements, where the first $N_{x} N_{y}$ values contain $\tilde{P}$, followed by $N_{x} N_{y}$ values of $c(\hat{\partial} \tilde{\boldsymbol{P}} / \partial z)$.

The solution of equation (3) is carried out in depth after $c(\hat{\partial} \tilde{\boldsymbol{P}} / \partial z)$ on the surface is calculated (see Kosloff and Baysal, 1983). The final depth section is accumulated according to the imaging condition

$$
P_{\mathrm{mig}}(x, y, z)=\sum_{\omega} \tilde{P}(x, y, z, \omega) .
$$

The generalized phase-shift method starts from the formal solution of equation (3) given by

$$
(\mathbf{V})_{z+d z}=e^{\mathbf{B} d z}(\mathbf{V})_{z}
$$

Equation (5) is evaluated by the expansion (Hamming, 1976)

$$
(\mathbf{V})_{z+d z}=e^{\mathbf{B} d z}(\mathbf{V})_{z} \simeq\left[\sum_{k=0}^{m} \alpha_{k} J_{k} R \underline{\mathbf{Q}}_{k}\left(\frac{\mathbf{B} d z}{R}\right)\right] \mathbf{V}_{z}
$$

where $\alpha_{0}=1, \alpha_{k}=2$ for $k>0 . R$ is chosen below to be larger than the eigenvalues of $\mathbf{B} d z$, and $J_{k}$ are Bessel functions of the first kind. $\mathbf{Q}_{k}$ are Chebychev matrix polynomials which are generated recursively by

$$
\underline{\mathbf{Q}}_{k+1} \mathbf{V}={\underline{\mathbf{Q}_{k-1}}}_{\mathbf{V}+2}\left(\frac{\mathbf{B} d z}{R}\right) \mathbf{Q}_{k} \mathbf{V}
$$

with $\mathbf{Q}_{0} \mathbf{V}=\mathbf{V}$ and $\mathbf{Q}_{1} \mathbf{V}=(\mathbf{B} d z / R) \mathbf{V}$.

For stable results, $R$ is defined with respect to the lowest velocity in the depth interval

$$
R=\frac{\omega d z}{C_{\min }(x, y, z=\text { constant })} .
$$

The expansion given in equation (6) is valid when the eigenvalues of $\mathbf{B} d z$ are purely imaginary, which requires the elimi- nation of the evanescent components from the solution (Kosloff and Kessler, 1987). For $k>R$, the expansion (6) converges exponentially and therefore can be truncated to a finite number of terms [ $m$ in equation (6)]. Since $\alpha$ is a constant and the Chebychev polynomials are bounded by unity, the Bessel functions serve as the criteria for truncating the expansion. Fquation (6) is evaluated for each frequency at all depths. The final section is accumulated according to equation (4).

\section{COMPUTATIONAL CONSIDERATIONS}

It was shown by Tal-Ezer (1986) and by Kosloff and Kessler (1987) that the integration technique used in the generalized phase-shift method is highly efficient when the integration step (depth step) is large. Since sampling considerations require a relatively small depth step (usually the choice will be $d z=$ $C_{\min } d t / 21$, a modified version of equation (6) is adopted:

$$
(\mathbf{V})_{z+\gamma d z}=e^{\mathbf{B} \gamma d z}(\mathbf{V})_{z} \simeq\left[\sum_{k=0}^{m} \alpha_{k} J_{k}(\gamma R) \mathbf{Q}_{k}\left(\frac{\mathbf{B} d z}{R}\right)\right] \mathbf{V}_{z},
$$

where $0<\gamma \leq 1$. The calculation of $\mathbf{Q}$ requires most of the computation time. Since $\mathbf{Q}$ is independent of $\gamma$, equation (9) can be used for large depth steps $d z$, and the required intermediate results $\gamma d z$ can be obtained with minor cost by generating additional sets of Bessel functions. In practice, the depth step should be defined by the resolution of the velocity function, and then the propagation in a large constant-velocity region can be accomplished without recalculating $\mathbf{Q}_{k}$. Not apparent from equation (9) is the dependence of the truncation index $m$ on the frequency. To determine the influence of the frequency band on the efficiency of the integration scheme, a synthetic example was tested. In this example the velocity was $2000 \mathrm{~m} / \mathrm{s}$; the time interval was $0.004 \mathrm{~s}$; and the sampling interval required for the depth section was $8.0 \mathrm{~m}$. Figure 1 represents the number of terms $(M)$ in the summation needed to achieve a specific accuracy ( 0.001 percent) as a function of the frequency. The number above each of the five lines indicates the number of sampling intervals (i.e., 1 is $8 \mathrm{~m}, 25$ is 200 $m$, etc.) in the integration step. From this figure, one can see the tradeoff between the depth step size and frequency band. In Table 1 an efficiency factor is given as a function of both depth step size and frequency band (a constant velocity of $2000 \mathrm{~m} / \mathrm{s}$ and a time interval of $0.004 \mathrm{~s}$ were used). The efficiency factor is the ratio between the number of compu-

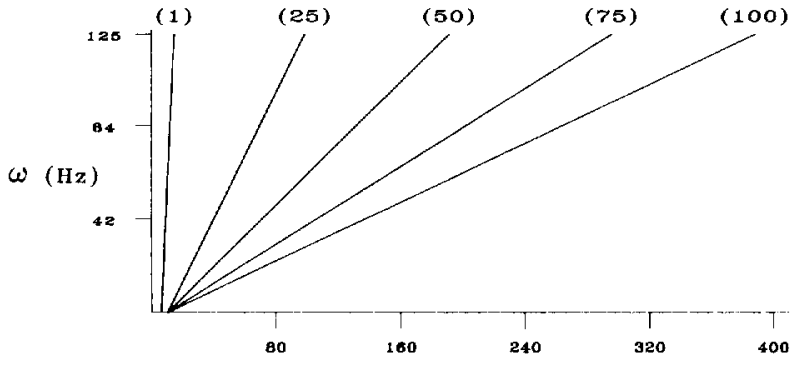

$M$

FIG. 1. The number of terms $M$ required to achieve 0.001 percent accuracy for five depth step sizes. $d z=8.0 \mathrm{~m}, d T=4.0$ $\mathrm{ms}$, and $c^{\circ}=2000 \mathrm{~m} / \mathrm{s}$. 
Table 1. Efficiency factor as a function of frequency and depth step size.

\begin{tabular}{|c|c|c|c|}
\hline $\begin{array}{c}\text { Step size } \\
\text { (m) }\end{array}$ & $32 \mathrm{~Hz}$ & $63 \mathrm{~Hz}$ & $125 \mathrm{~Hz}$ \\
\hline 8.0 & 1.0 & 1.0 & 1.0 \\
\hline 200.0 & 6.8 & 5.2 & 3.9 \\
\hline 400.0 & 8.6 & 6.1 & 4.4 \\
\hline 600.0 & 9.5 & 6.6 & 4.6 \\
\hline 800.0 & 10.2 & 6.9 & 4.8 \\
\hline
\end{tabular}

tations needed to extrapolate to a certain depth with a step size of $8 \mathrm{~m}$ and the number of computations needed to extrapolate to the same depth with larger depth steps. The frequency in this table is the highest frequency used in equation (4). Since the accuracy of the method is not affected by the step size, the table indicates that a large depth step should be used whenever possible. However, when higher frequencies are included in the summation, the effectiveness of larger depth steps is less significant.

In the generalized phase-shift method, the spatial derivatives are calculated by FFTs (Gazdag, 1980; Kosloff and Baysal, 1983). The use of FFTs makes the calculation of matrix $\mathbf{B}$ [see equation (3)] the most time-consuming part in the algorithm. When the velocity function consists of only shallow dipping interfaces, a slightly modified version of equation (1).

$$
c^{2} \frac{\partial^{2} \tilde{\boldsymbol{P}}}{\partial x^{2}}+c^{2} \frac{\partial^{2} \tilde{P}}{\partial y^{2}}+c \frac{\partial}{\partial z}\left(c \frac{\partial \tilde{P}}{\partial z}\right)=-\omega^{2} \tilde{P}
$$

can save many computations. This version of the nonreflecting wave equation gives an impedance match only in the vertical (z) direction (Baysal et al., 1984). In this case, the number of FFT operations needed to compute $\mathbf{B}$ is half the number of operations needed in the general case. The calculations of the space derivative terms in $\mathbf{B}$ require only a single forward and a single inverse FFT. When the general form [equation (2)] is applied, each space derivative computation requires two forward and two inverse FFTs, since multiplication by $c$ has to be performed in between.

To process field data, the use of FFTs may require the addition of null traces to the data in order to match the FFT operator size. Since the addition must be done in both the $x$ and $y$ directions, it can result in a significant increase in the data-set size. In this study a mixed-radix algorithm to calculate FFTs was used (Temperton, 1983). This algorithm allows more flexibility in the choice of the grid length compared to the traditional radix-two algorithms and therefore the addition of zero traces can be minimized. Implementation of the algorithm on a vector computer does not show degradation in performance compared to the radix-two algorithms. Furthermore, the ability to compute odd-base FFTs may eliminate some performance degradation associated with memory bank conflicts on most vector computers.

\section{COMPUTER IMPLEMENTATION}

Three-dimensional depth migration of a typical data set requires an amount of memory generally exceeding the central memory size of available computers. Therefore, an out-of-core scheme must be employed. The major issues in the design of very large out-of-core algorithms are, first, the large amount of $\mathrm{I} / \mathrm{O}$ activity and, second, computation time.

We shall illustrate the $\mathrm{I} / \mathrm{O}$ problem with an example. In this example, the input stacked data set contains 360 lines with 375 traces in each line. The time increment is $0.004 \mathrm{~s}$ and each trace is $6.0 \mathrm{~s}$ long (1500 samples). The size of this data set is 202.5 million words ( $\mathrm{MW}$ ). The desired depth section contains 1000 samples at $8 \mathrm{~m}$ intervals and requires $135 \mathrm{MW}$ of storage. When computations are done in the frequency domain, an appreciable reduction in the input data-set size can be achieved. If for example we use $66.5 \mathrm{~Hz}$ as the high-cut frequency, only 400 frequencies are needed for the computations $(125 \mathrm{~Hz}$, the alias frequency in this case, will require $750 \mathrm{fre}$ quencies). $\tilde{P}(x, y, \omega)$ is kept real and becomes an array of $375 \times 360 \times 400$ or $54 \mathrm{MW}$. Even though the algorithm requires both $\tilde{P}$ and $c(\hat{C} \tilde{P} / \hat{O} z)$, the total amount of data is still only about half the initial data-set size.

The fact that the above integration scheme is a one-step scheme allows the arrays that contain the transformed pressure field and its derivative to be updated at each depth step with no extra memory requirement. With a configuration of 8 MW of central memory, an out-of-core solution must be implemented. This example requires approximately 1.73 gigabytes of data (a Cray word consists of eight bytes) to be transferred to and from the central memory at each depth step. A proper organization of the data $[\tilde{P}$ and $c(\delta \tilde{P} / \partial z)]$ on a high-speed storage device (currently capable of transferring more than 2 billion bytes per second) makes $\mathrm{I} / \mathrm{O}$ time almost negligible and the algorithm becomes CPU bound.

The migration algorithm requires an enormous amount of computation time, even for very fast vector computers. A shortened elapse time for large out-of-core applications can be achieved only if a high degree of parallelism occurs in the code (Larson, 1984). In the algorithm just described, the migration is carried out in the frequency domain. The downward continuation of each frequency is totally independent from the continuation of other frequencies. Parallelism can therefore be implemented at a very high level in the code. With the use of the Cray-XMP/48 (four processors), the speedup factor that we obtained was 3.75 . It should be pointed out that, due to the high transfer rate of the storage device, the $\mathrm{I} / \mathrm{O}$ operations were done sequentially. The same algorithm can be adopted with slower storage devices by overlapping the intensive $1 / O$ activity with the computations, provided that $\mathrm{I} / \mathrm{O}$ operations can be performed inside the parallel region of the code.

The computations for the above example require $80 \mathrm{~s}$ of elapsed or total time to downward extrapolate all frequencies a single depth step. If, for example, a velocity function is given at a vertical interval of $50 \mathrm{~m}$, the whole depth section $(8000 \mathrm{~m})$ can be calculated in 3.5 hours. With input traces of the same length and for the same frequency band, the largest data set that has been migrated consisted of 750 by 810 traces. It required the use of a $512 \mathrm{MW}$ solid-state storage device. Wallclock time (using four CPUs) in this case was about four times longer than the above example, indicating that with such a storage device, the algorithm is totally CPU bound.

\section{COMPARISON WITH OTHER MIGRATION METHODS}

We divide the comparison with other migration methods into two parts. First, the proposed depth extrapolation is 
Table 2. 1/O requirements - comparison between time and depth extrapolation.

\begin{tabular}{lcccc}
\hline & $\begin{array}{c}\text { Reverse-time } \\
\text { migration }\end{array}$ & & $\begin{array}{c}\text { Depth } \\
\text { extrapolation }\end{array}$ & $\begin{array}{c}\text { Ratio } \\
\text { (depth:time) }\end{array}$ \\
\cline { 2 - 2 } $\begin{array}{c}\text { Fast } \\
\text { storage }\end{array}$ & $405.0 \mathrm{MW}$ & & $108 \mathrm{MW}$ & $1: 3.75$ \\
$\begin{array}{c}\text { Slow } \\
\text { storage }\end{array}$ & $607.5 \mathrm{MW}$ & & $270 \mathrm{MW}$ & $1: 2.25$ \\
$\begin{array}{c}\text { Total I/O } \\
\text { activity }\end{array}$ & $3645 \mathrm{GW}$ & $216 \mathrm{GW}$ & $1: 16.87$ \\
\hline
\end{tabular}

compared to time extrapolation (i.e., reverse-time migration), and, then, the method is compared to other space-frequency algorithms.

We now consider the example given in the previous paragraph. For reverse-time migration, a finite-difference scheme (fourth order in space and second order in time) was chosen. We assume that horizontal sampling is small enough to avoid aliasing. With a maximum velocity of $6000 \mathrm{~m} / \mathrm{s}$, stability requirements dictate a time increment of $0.00133 \mathrm{~s}$ for the finite differencing. The original time section was resampled to three times the original sampling density and occupied $607.5 \mathrm{MW}$ of storage, although this could have been cut to about $203 \mathrm{MW}$ by interpolating the data only at the points where they were needed for computation. An explicit solution scheme required three global arrays (135 MW each) for the pressure, time derivative of the pressure, and the velocity. These latter arrays needed to be accessed and restored for each of the 4500 time steps. The above requirements are compared to the worst case for the generalized phase-shift method, where the integration step is the depth sampling interval $(8 \mathrm{~m})$. Table 2 summarizes the $\mathrm{I} / \mathrm{O}$ requirements of both methods. $\mathrm{A}$ fast storage device is needed for data repeatedly accessed and restored, and a slow device can be used for data that are accessed or stored once. Assuming an out-of-core scheme, total $1 / O$ activity is the number of words that are accessed/restored from/to the fast storage device during the migration. For example, $3645 \mathrm{MW}$ is 405 (three arrays of $135 \mathrm{MW}$ each) times 4500 (number of time steps) times 2 (for access and restore). The table indicates that for reasonable size data sets, the feasibility of migrating the data with the reverse-time method on existing hardware is questionable. Furthermore, any increase in the number of depth samples or maximum velocity results in a significant growth in fast storage space and total $\mathrm{I} / \mathrm{O}$ operations with the reverse-time method. In contrast, the depth extrapolation scheme we presented needs fewer $\mathrm{I} / \mathrm{O}$ operations when large integration steps are used. An increase in the number of depth (and velocity) samples changes only the slow storage requirements. Trivial increase of $\mathrm{I} / \mathrm{O}$ activity is introduced in this case, since only one velocity slice is accessed and one depth slice is stored in every depth step, and fast storage space remains the same. In addition to the $\mathrm{I} / \mathrm{O}$ problem of the reversetime method, a significant increase in the amount of computation should be expected because resampling the data to a smaller time increment is usually required.

The use of the pseudospectral method for reverse-time migration is even more expensive than the simple finite-difference scheme, mainly because of stronger stability restrictions, resulting in a smaller time increment for the time-stepping procedure. In this case, $\mathrm{I} / \mathrm{O}$ and computational power requirements are even harder to meet.

In the case of poststack migration, we assume that the data have no multiples and correct amplitude treatment is not essential. When the continuity of $\partial P / \partial z$ (which enables correct amplitude treatment) and the presence of downgoing waves (for correct reconstruction of multiples) can be ignored, a oneway wave equation can be used for depth extrapolation.

The algorithm chosen here, although presented with a twoway wave equation, can be implemented with other wave equations that can be expressed in the general form of equation (3). With the present scheme, a one-way wave equation involves less computation. Computational efficiency in this case depends on dip limitations, the lateral velocity variations allowed, and, mainly, the choice of operator for space derivative calculations. In terms of memory and storage requirements, there is no need for the $\partial P / \partial z$ array when a one-way equation is used. The use of a two-way equation, on the other hand, eliminates the necessity for complex arrays and complex arithmetic in the solution scheme. However, if the downgoing waves are to be eliminated by filtering out components with negative vertical wavenumbers at the end of the two-way wave-equation migration (Kosloff and Baysal, 1983), complex arrays must be used during the migration. The use of a twoway nonreflecting wave equation for migration of stacked data does not require the elimination of downgoing waves, and complex arrays and complex arithmetic can be avoided.

\section{SYNTHETIC EXAMPLE}

To check the accuracy of the method, a 3-D synthetic time section was generated using the exploding-reflector option, based on a nonreflecting wave equation (Reshef et al., 1988). The configuration and the physical parameters of the threelayer model are shown in Figure 2a. This figure represents a 2-D cross-section, perpendicular to the $y$ direction, along which the medium is invariant. A $3-\mathrm{D}$ grid (128 nodes on each axis) was used for the modeling with a grid spacing of $8.0 \mathrm{~m}$ in all three directions. Quantization of the structure into this grid is shown in Figure $2 b$. The high-cut frequency of the Rickertype source wavelet was $60 \mathrm{~Hz}$. Figure 3 represents one of the 2-D time sections obtained from the modeling.

Next, a depth migration, based on equation (2), was performed. The required sampling interval for the depth section was $2.0 \mathrm{~m}$. Since the velocity function that we used for the migration was the same as that used in the forward modeling (i.e., sampled at $8 \mathrm{~m}$ intervals), the factor $\gamma$ [see equation (9)] was 0.25 . Figure 4 shows one of the 2-D depth sections obtained from the migration. Comparison between this figure and the original model (Figure $2 b$ ) shows that the reflectors were correctly reconstructed by the migration (this includes the diffractions beneath the upper reflector, caused by stairstepping in the original model).

\section{FIELD DATA EXAMPLE}

Figures 5-8 show application of the method to a small 3-D land data volume. The stacked data consisted of 65 lines with 113 traces in each line. Every trace contained 1500 samples at 


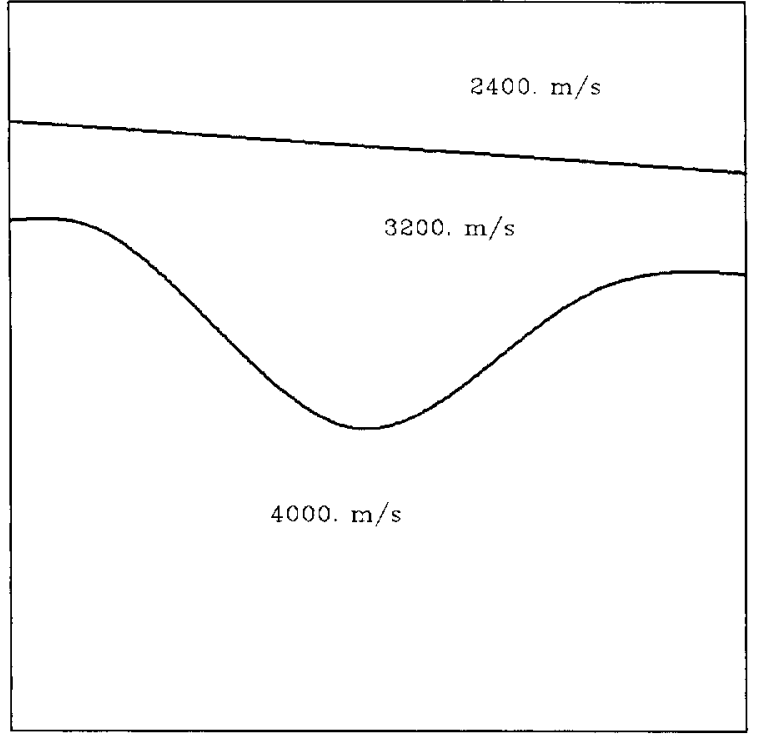

$1024 \mathrm{~m}$

(a)

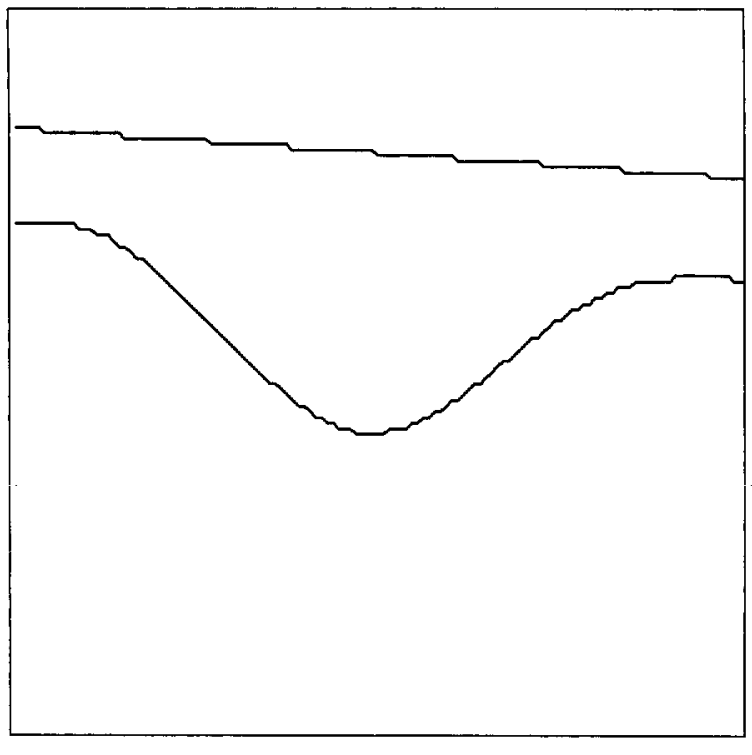

(b)

Fig. 2. (a) Physical parameters and model configuration for the synthetic example. (b) Quantization of the model into the computed grid.

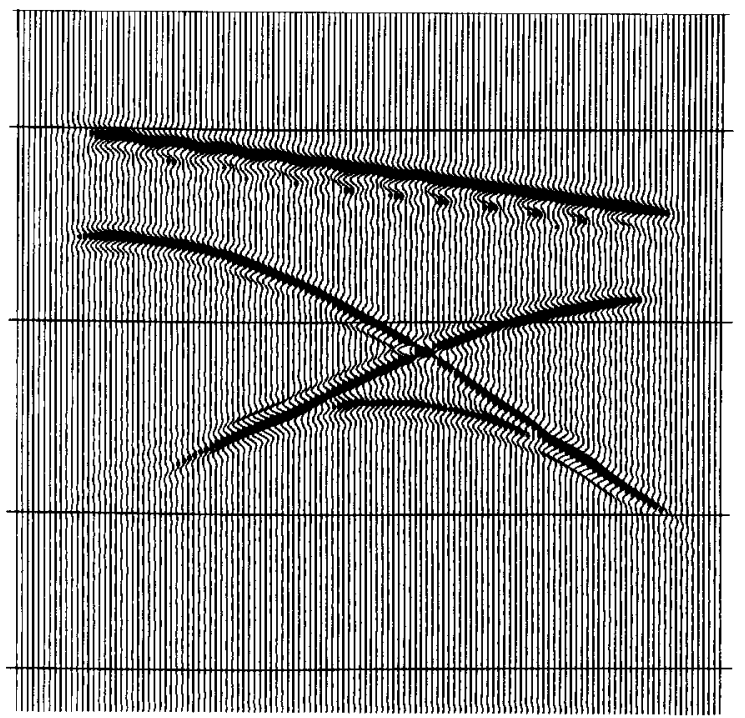

0.17

FIG. 3. Synthetic 2-D time section.

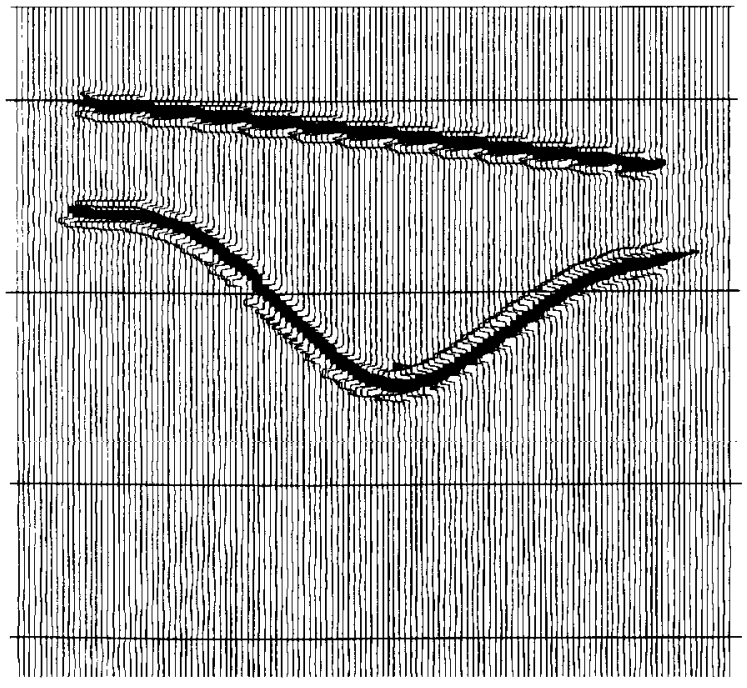

200.

soo.

750.

FIG. 4. Synthetic example---depth migration results.
0.51

0.69 


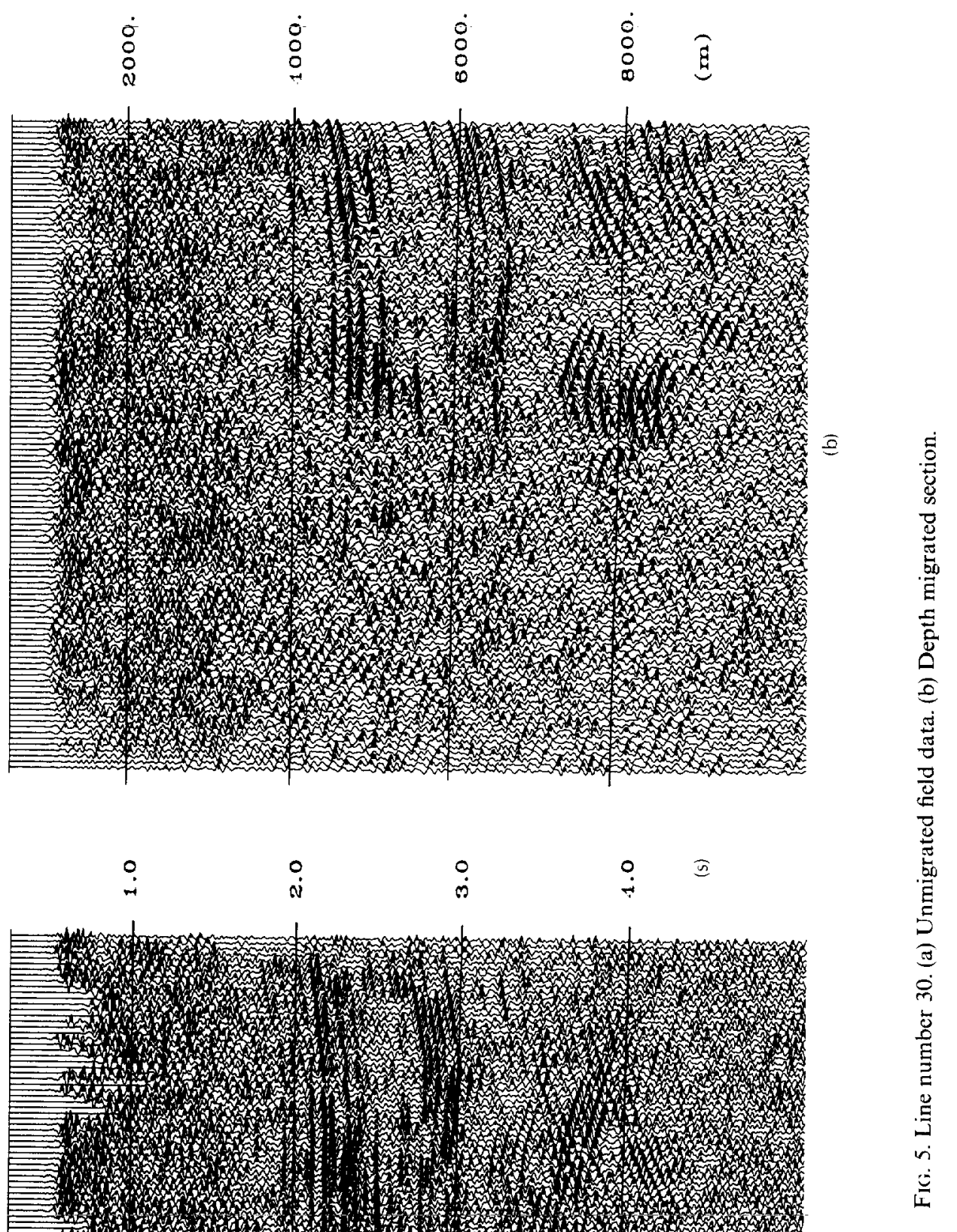




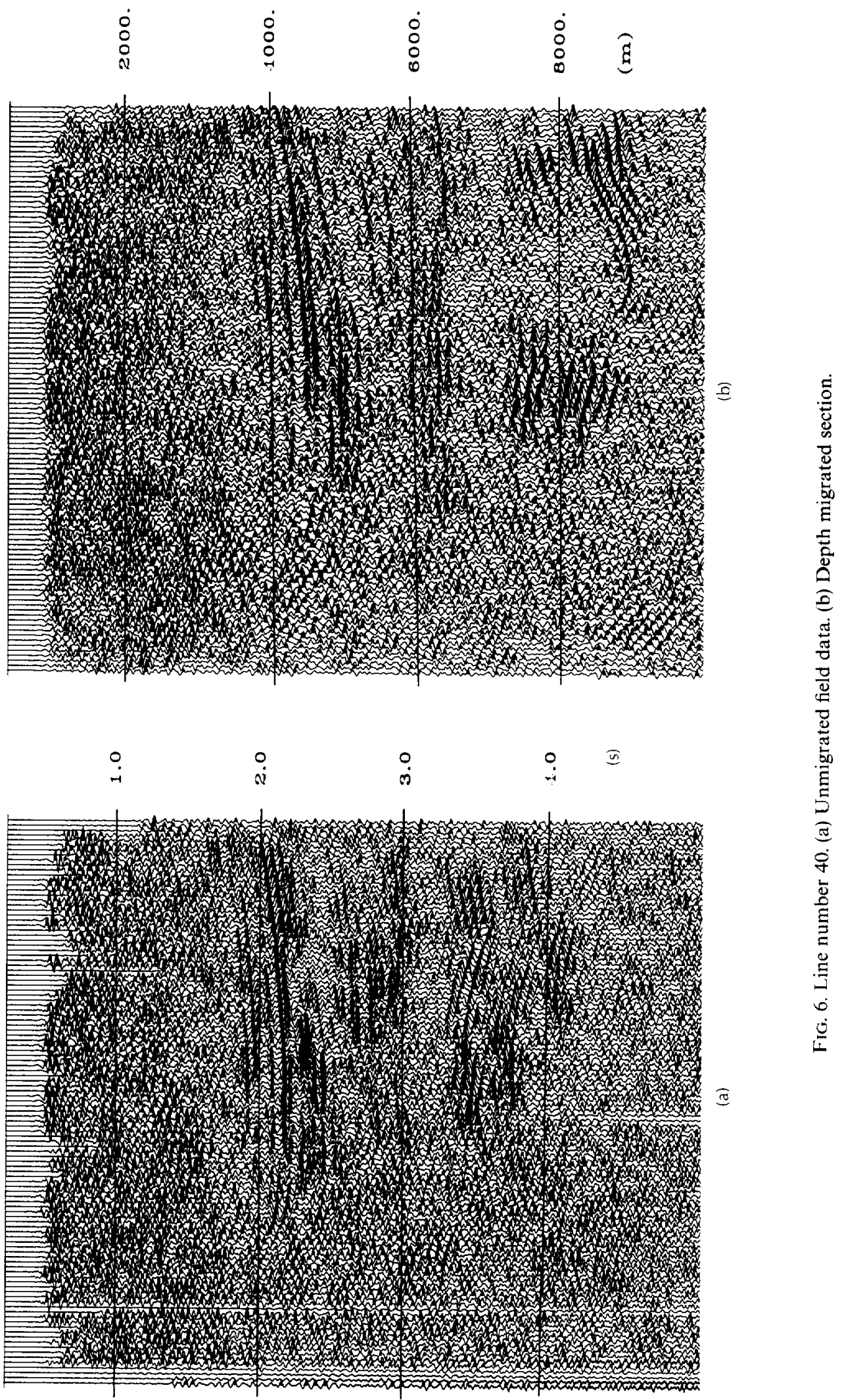




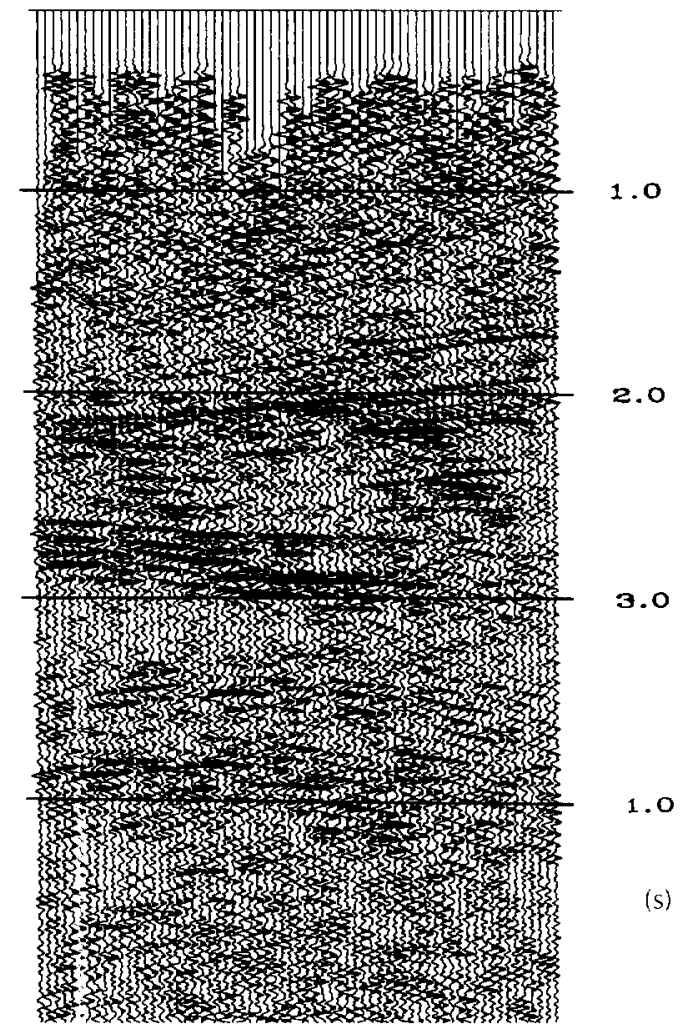

(a)

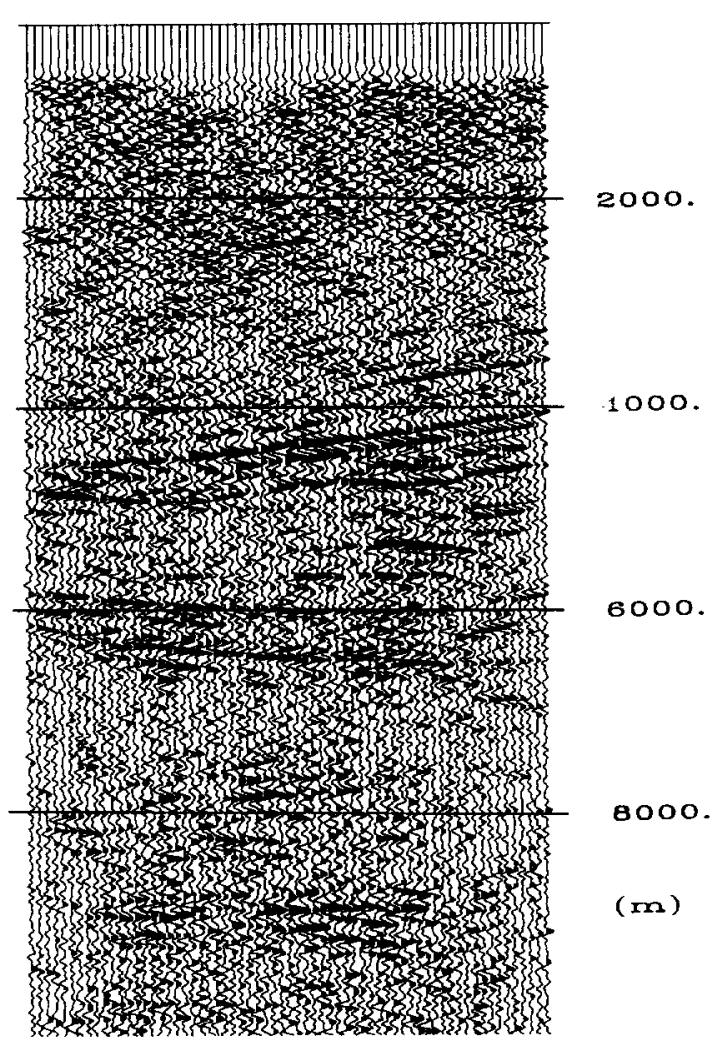

(b)

Fic. 7. Cross-line number 90. (a) Unmigrated field data. (b) Depth migrated section.

a sample rate of $0.004 \mathrm{~s}$. The horizontal distance between the traces was $100 \mathrm{~m}$ in both $x$ and $y$ directions. The sampling interval of the output depth section was $6.7 \mathrm{~m}$ and the migration was continued to a depth of $9.6 \mathrm{~km}$. Since the velocity function provided consisted of a few flat interfaces, a depth interval of $167 \mathrm{~m}$ was used for the calculations. The use of FF" Ts for the computations of the spatial derivatives required the addition of ten lines and seven traces per line for a total of $120 \times 75$ traces. The highest frequency used in the migration was $50 \mathrm{~Hz}$. The total time required to migrate the data set was 25 minutes.

Figures 5 and 6 represent the unmigrated time section (a) and the corresponding depth migrated section (b) for lines 30 and 40, respectively. Most of the clearly defined events appear on the right-hand sides of the sections. The upper $3 \mathrm{~s}$ show mainly flat reflectors that are vertically stretched by the migration. Much better definition of the structure occurs near 8000 $\mathrm{m}$ in the depth sections, where reflector shapes are distorted in the time sections (near $3.5 \mathrm{~s}$ ). A cross-line comparison is shown in Figure 7 , where (a) is the unmigrated time section of cross line 90 and (b) is the corresponding depth migrated section. In general, the comparison between the time sections and the depth sections shows that the migration method can be used successfully with field data.

To compare results with the reverse-time method, the same data set was migrated with both methods. Parameters for the migration were the same as for the depth extrapolation. Figure 8 a represents part of depth line 50 , as obtained by the generalized phase-shift migration. A corresponding result was obtained by the reverse-time migration (Figure $8 \mathrm{~b}$ ). Differences in results are attributed to the use of different space derivative operators (fourth-order differencing in the reversetime migration and Fourier in the generalized phase shift).

\section{CONCLUSIONS}

In this work we have demonstrated that the use of advanced supercomputers, together with a proper algorithm, makes the implementation of an accurate 3-D depth migration feasible. With high-speed storage devices, large out-of-core problems can become CPU bound. Since the described algorithm is highly parallel, we believe that with future increases in CPU speed and with more CPUs, the processing time for the proposed migration method will be significantly reduced.

This present work suggests that depth extrapolation is computationally more efficient than reverse-time migration. Because accuracy requirements can be satisfied by depth or time extrapolation, the much smaller $\mathrm{I} / \mathrm{O}$ requirements and the computational flexibility of the depth extrapolation should make it the preferable method. In fact, with existing hardware, reverse-time migration cannot be performed on realistic size data sets in a reasonable amount of time. The only drawback of the depth extrapolation remains the requirement of eliminating the evanescent energy components from the solution.

Depth extrapolation is the basis for many iterative migration and inversion algorithms. Because of the ability to relate 


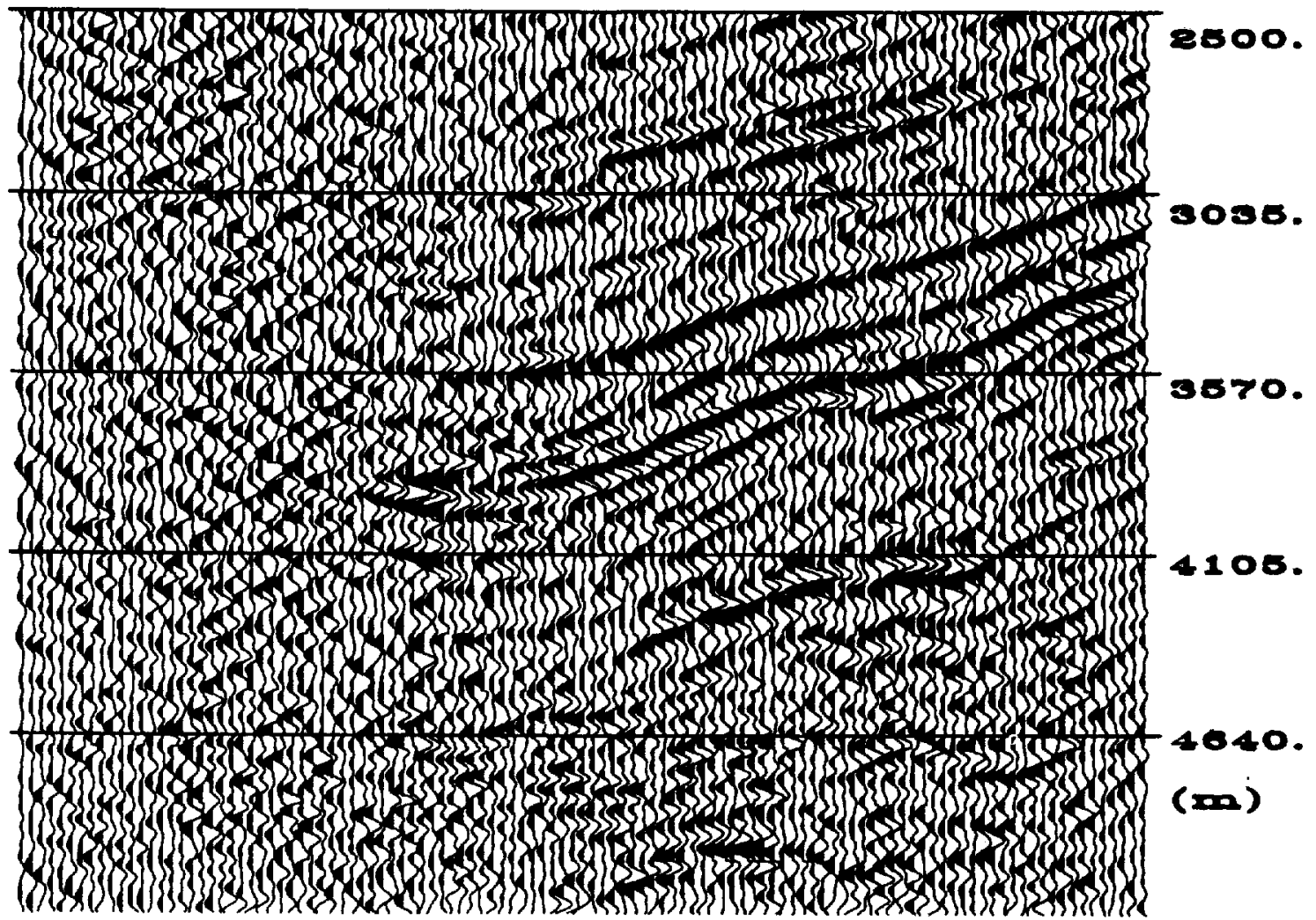

(a)

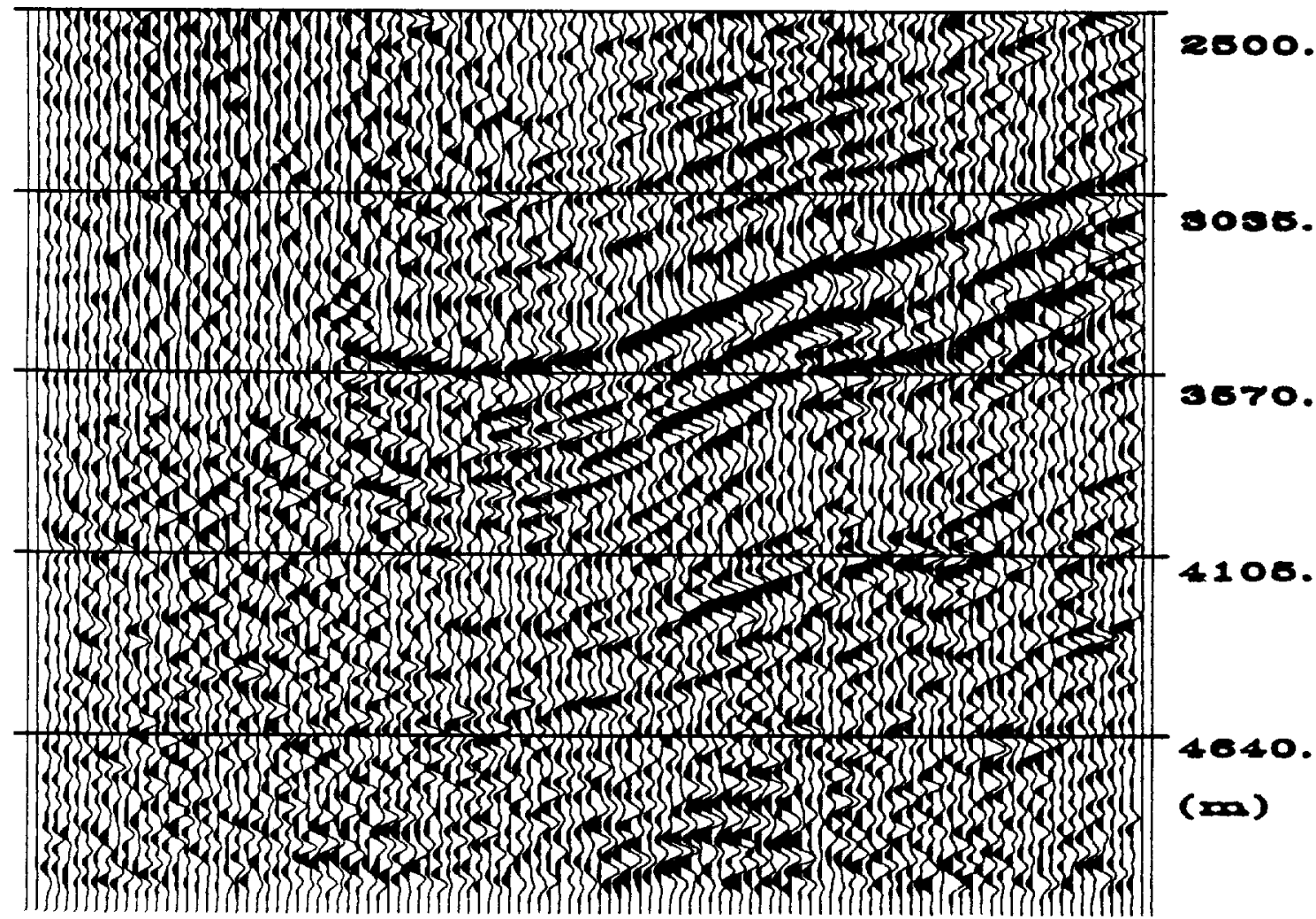

(b) 
the number of computations to the complexity of the velocity function, the technique presented above should be considered for such algorithms.

\section{ACKNOWLEDGMENTS}

This study was partially supported by the Pikovski-Valatzi foundation. The 3-D data set (Figures 5-8) appears by courtesy of Phillips Petroleum Co.

\section{REFERENCES}

Baysal, E., Kostoff, D., and Sherwood, J. W. C., 1984, A two-way nonreflecting wave equation: Geophysics, 49, 132-141.

Berkhou1, A. J., 1981, Wave-field extrapolation techniques in seismic migration, a tutorial: Geophysics, 46, 1638-1656.

Gazdag. J., 1980, Wave equation migration with the accurate space derivative method: Geophys. Prosp., 28, 60-70.

Hamming. R. W., 1976. Numerical methods for scientists and engineers: McGraw-Hill Book Co.

Kosloff, D., and Baysal, E., 1983, Migration with the full acoustic wave equation: Geophysies, 48, 677-687.
Kosloff, D., and Kessler, D., 1987, Accurate depth migration by a generalized phase-shift method: Geophysics, 52, 1074-1084.

Larson, J. L., 1984, Multitasking on the Cray-XMP/2 multiprocessor: Inst. Electr. Electron. Eng. computer, 17, 62-69.

Reshef, M., Kosloff, D., Edwards, M., and Hsiung, C., 1988, Threedimensional acoustic modeling by the Fourier method: Geophysics, 53. $1175-1183$

Stolt, R. H., 1978, Migration by Fourier transform: Geophysics, 43, $23-48$.

Tal-Ezer, H., 1986, Spectral methods in time for hyperbolic problems: Soc. Industr. Appl. Math. J. on Num. Anal, 23, $11-26$.

Tarantola, A.. Jobert, G., Trezeguet, D., and Denelle, E., 1988, The non-linear inversion of seismic waveforms can be performed either by time extrapolation or by depth extrapolation: Geophys. Prosp., 36, $383+416$

Temperton. C.. 1983. Self sorting mixed radix Fast Fourier Transforms: Jour. Comp. Phys., 52, 1-23.

Wapenaar, C. P. A., and Berkhout, A. J., 1986, Wave field extrapolation techniques for inhomogeneous media which include critical angle events. Part II: methods using the two-way wave equation: Geophys. Prosp., 34, 147-179.

Whitmore. N. D., 1983, lterative depth migration by backward time propagation: 53rd Ann. Internat. Mtg., Soc. Expl. Geophys., Expanded Abstracts, 382-385. 